\title{
A New Evaluation Method for Measuring the Effectiveness of Mobile Applications: A Mathematical Modelling Approach
}

\author{
Amalina F. A. Fadzlah \\ Department of Computer Science, Faculty of Science and Defence Technology, Universiti Pertahanan Nasional \\ Malaysia, Kem Perdana Sg Besi, Kuala Lumpur, Malaysia \\ Corresponding author. Email: amalina.farhi@upnm.edu.my \\ Manuscript submitted March 10, 2017; accepted July 8, 2017. \\ doi: $10.17706 / j c p \cdot 13 \cdot 3 \cdot 360-370$
}

\begin{abstract}
This paper presents a mathematical modelling approach for measuring the effectiveness of mobile applications. This research considers specifically the measurements of effectiveness parameters useful to express and estimate the overall effectiveness of mobile applications usage. As a result, a new and simple mathematical-based evaluation model for measuring the effectiveness of mobile applications, namely Mobile Applications Effectiveness Measurement Model (MoAEMM), was established. The proposed method integrates 23 metrics and 8 attributes into three criterions for evaluating mobile applications effectiveness including accurateness, readiness, and completeness. The model presented not only reveals the effectiveness measurements for assessing the mobile applications, but also provide a better understanding of the relationship between these measures. In addition, the findings can be employed as a new and concrete evaluation method with which to assess the effectiveness of mobile applications usage from the perspective of mathematical-based modelling approach.
\end{abstract}

Key words: Evaluation method, effectiveness measurement, mathematical approach, measurement model, mobile applications.

\section{Introduction}

Effectiveness, in general is described as the capability of doing something successfully with which targeted objectives are achieved. Other definition regards to the extent to which the targeted problems are solved correctly. Both definitions were supported by many researchers in regards to the concept of usability, that relates effectiveness as a measure of successfulness and correctness with which the specified goals or subgoals of using particular system can be achieved [1]. Other researchers mentioned effectiveness reflected by the quality of solutions and error rates made by users in order to achieve certain goals [2]. The international standards also provide a broader guidance on effectiveness and define it as the accuracy and completeness with which specified users can achieve specified goals by using specified equipment in particular environments [3]. Most of these definitions focused on the devices and applications of conventional computing environment. As innovations and new technology have emerged, there is a need to investigate the term effectiveness in more advanced perspectives [4].

Nowadays, mobile devices are finding their way into anyone, anytime, anywhere and anything computing environments [5]. This is due to the devices significant advantages provided to its users, in terms of affordability, portability, accessibility and functionality [6]. The variety of capabilities of these devices has led 
to tremendous expansion of mobile applications being designed and developed over the past few years [7]. Thus, challenged the design and development of mobile applications towards superior quality, an important one is being effective, in order to compete in the market place [8]. However, due to the hardware and software constraints (i.e. small screen size, data entry problems, connectivity issues, and varying display resolutions), there are many aspects to consider for designing and developing effective mobile applications [9]. Such aspect that needs to be taken into account is a number of evaluation procedures for assessing and measuring the effectiveness of mobile applications among its respective users [10].

Overall, the study of the phenomena in the field of evaluating mobile applications is highly driven by quality perspective and concentrates primarily on producing useful and usable products rather than reflecting on measuring the usage effectiveness in detail. For examples, quality models developed, described effectiveness as the key factor in the development of successful mobile-based software applications [11]. Other researchers continued the study with the development of software certification framework and models for evaluating mobile applications effectiveness [12]. Meanwhile, Fadzlah et al. proposed the concept of effectiveness in assessing the usability of mobile applications usage [13]. Yet, only a few viewed as independent models which lay down general measures and measurements to demonstrate the evaluation of mobile applications effectiveness [14]. Most of them focused on evaluating the usability of very specific types and usages of mobile applications [15].

There are many ways in which evaluations can be described [16]. One of the current trends in evaluating mobile applications is using a mathematical modelling approach [17]. Mathematical modelling approach is the art of translating problems from an application area into tractable mathematical formulations whose theoretical and numerical analysis provides insight, answers, and guidance useful for the originating application [18]. There are several works done on evaluating mobile applications using a mathematical modelling approach [19]. However, none of the researchers concentrated on developing a mathematical model for assessing and measuring mobile applications effectiveness, in general. Due to this reason, the strong demand for developing a new evaluation method for measuring mobile applications effectiveness via mathematical modelling approach thus burgeoning.

In this paper, a new evaluation method for measuring the effectiveness of mobile applications was proposed, focusing on measuring the mobile applications usage with mathematical modelling approach. This research considers specifically the measurements of effectiveness parameters useful to express and estimate the overall effectiveness of mobile applications usage. As a result, a new and simple mathematical-based evaluation model for measuring the effectiveness of mobile applications, namely Mobile Applications Effectiveness Measurement Model (MoAEMM), was established. This model extended the hierarchical conceptual and empirical relationship-driven framework developed by Fadzlah [20]. This framework brings together different measures in different hierarchy levels. Each level represents interaction with other level and impacts one another to measure the desired mobile applications usage.

This can be explained as either none, one or more metrics could represent a single attribute. The combination of these metrics could be represented as the measures that contributed to only one attribute. Further, the combination of these attributes could be represented as the measures that contributed to only one criterion. Finally, these criterions are used to support in the calculation of the factor that can be concluded as directly affected the final measure of mobile applications usage. This is the case at every level in which could be represented as an M-1 relationship. For example, metric $\mathrm{M}_{1} \ldots \mathrm{Mn}$ are the input to attribute $\mathrm{A}_{1}$ and criterion $C_{1}$ is an output for the attribute $A_{1}$. Consider if the value of metric $M_{1}, M_{2}, \ldots, M n-1$ or $M_{n}$ increases so as the value of attribute $A_{1}$ and criterion $C_{1}$. Again, if the value of metric $M_{1}, M_{2}, \ldots, M n-1$ or $M_{n}$ decreases so as the value of attribute $A_{1}$ and criterion $C_{1}$. Fig. 1 shows the framework consisted of criterions, attributes and metrics for measuring mobile applications effectiveness. 


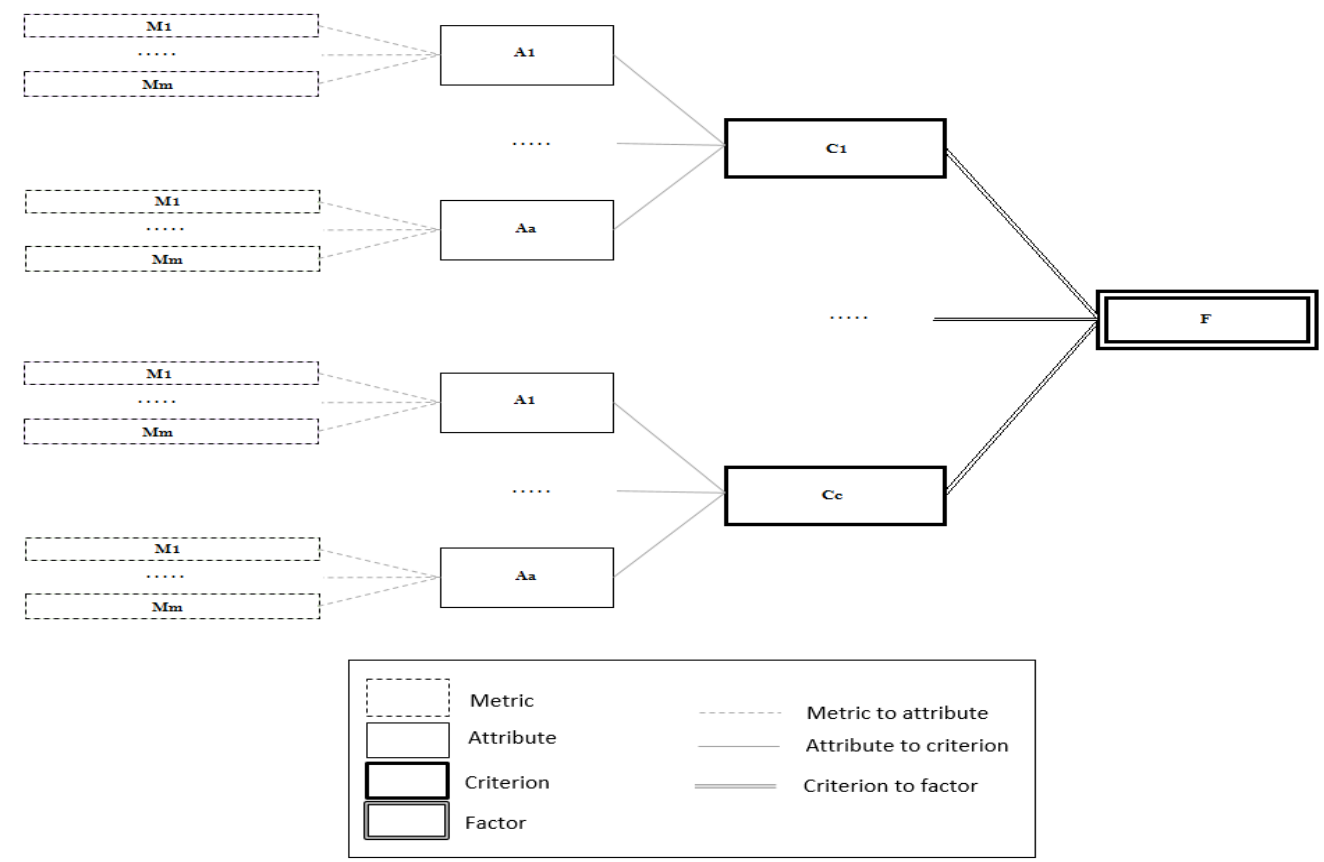

Fig. 1. Hierarchical level of metric, attribute and criterion for measuring effectiveness.

\section{Materials and Methods}

Table 1. Criterion, Attribute and Metrics of Effectiveness

\begin{tabular}{|c|c|c|}
\hline Criterion & Attribute & Metric \\
\hline \multirow[t]{9}{*}{ Accurateness } & \multirow[t]{3}{*}{ Action Adequacy } & Error Prevented depends on the number of errors prevented correctly \\
\hline & & Error Detected depends on the number of errors detected correctly \\
\hline & & Failure Recovered depends on the number of failures recovered correctly \\
\hline & \multirow[t]{3}{*}{ Literacy Error } & Text Read depends on the number of texts read correctly \\
\hline & & Syllable Spoken depends on the number of syllables spoken correctly \\
\hline & & Word Written depends on the number of words written correctly \\
\hline & \multirow[t]{3}{*}{ Spatial Accuracy } & Target Identified depends on the number of objects identified correctly \\
\hline & & Input Entered depends on the number of inputs entered correctly \\
\hline & & Page Navigated depends on the number of pages navigated correctly \\
\hline \multirow[t]{10}{*}{ Readiness } & \multirow{4}{*}{$\begin{array}{l}\text { Knowledge } \\
\text { Acquisition }\end{array}$} & Skill Integrated depends on the number of skills integrated correctly \\
\hline & & Problem Solved depends on the number of problems solved correctly \\
\hline & & $\begin{array}{l}\text { Meaning Comprehended depends on the number of meanings comprehended } \\
\text { correctly }\end{array}$ \\
\hline & & Reason Explained depends on the number of reasons explained correctly \\
\hline & \multirow{3}{*}{$\begin{array}{l}\text { Individual } \\
\text { Interpretation }\end{array}$} & Answer Supplied depends on the number of answers supplied correctly \\
\hline & & Example Provided depends on the number of examples provided correctly \\
\hline & & $\begin{array}{l}\text { Question Understood depends on the number of questions understood } \\
\text { correctly }\end{array}$ \\
\hline & \multirow[t]{3}{*}{ Interface Acceptance } & $\begin{array}{l}\text { Information Memorized depends on the amount of information memorized } \\
\text { correctly }\end{array}$ \\
\hline & & $\begin{array}{l}\text { Representation Recalled depends on the number of representations recalled } \\
\text { correctly }\end{array}$ \\
\hline & & Layout Recognized depends on the number of layouts recognized correctly \\
\hline \multirow[t]{4}{*}{ Completeness } & \multirow[t]{2}{*}{$\begin{array}{l}\text { Goal } \\
\text { Accomplishment }\end{array}$} & $\begin{array}{l}\text { Task Completed depends on the number of tasks completed within session or } \\
\text { treatment }\end{array}$ \\
\hline & & $\begin{array}{l}\text { Task Completed }(\mathrm{T}) \text { depends on the number of tasks completed within an } \\
\text { allotted time }\end{array}$ \\
\hline & \multirow[t]{2}{*}{ User Willingness } & $\begin{array}{l}\text { Self Motivated depends on the number of users motivated to complete task } \\
\text { within session or treatment }\end{array}$ \\
\hline & & $\begin{array}{l}\text { Self Motivated }(\mathrm{T}) \text { depends on the number of users motivated to complete } \\
\text { task within an allotted time }\end{array}$ \\
\hline
\end{tabular}


The main purpose of this study was to develop a model describing a mathematical-based evaluation technique for assessing the effectiveness of mobile applications. As a result, a total number of 23 metrics and 8 attributes and 3 criterions were identified having associated towards measuring the effectiveness of mobile applications usage. The definition of each effectiveness measure as well as the classification of these effectiveness measures according to its corresponding hierarchy levels is as shown in Table 1 above.

\section{Results and Findings}

\subsection{Effectiveness Measurement Scale}

Table 2. Effectiveness Measurement Items

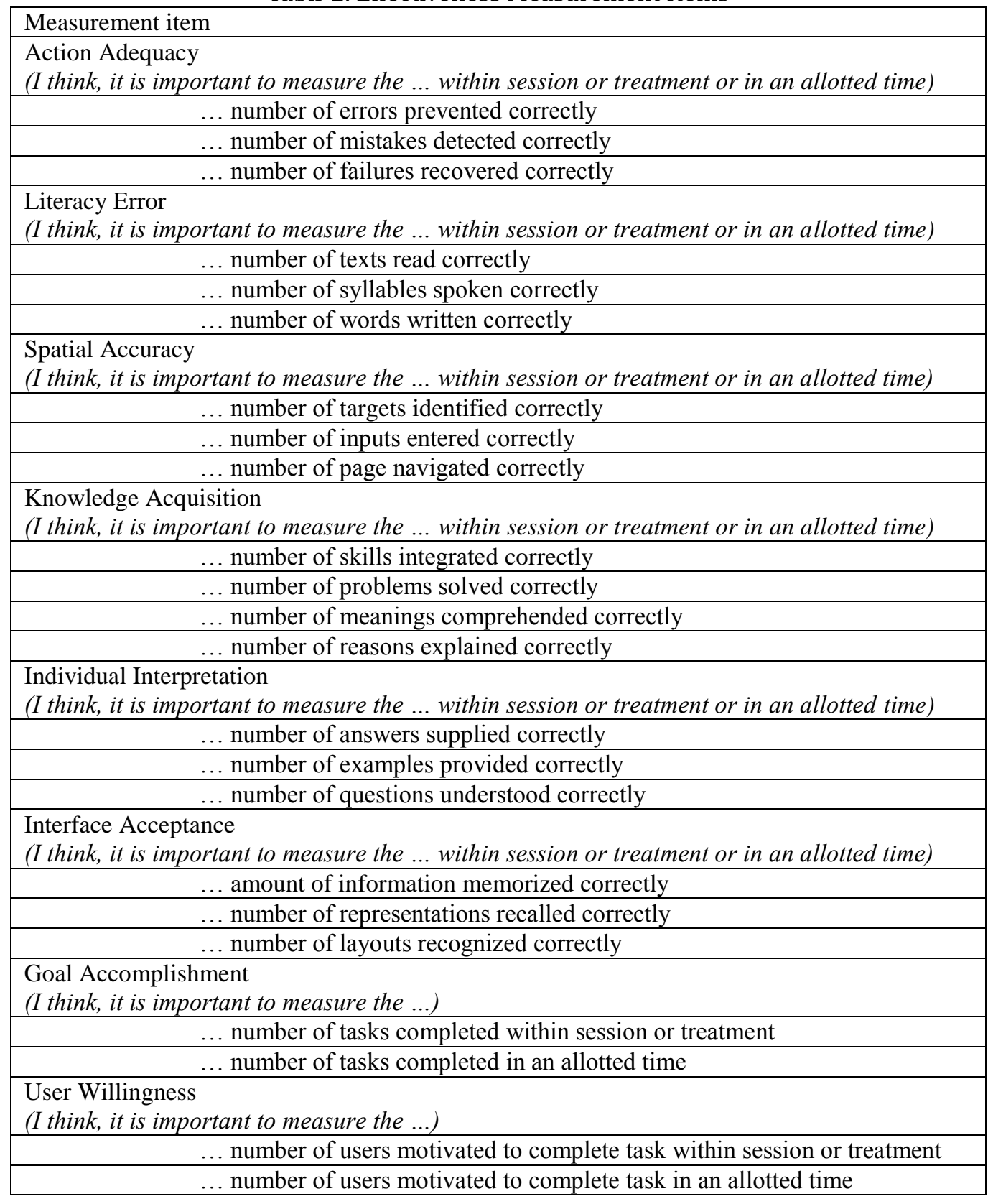

The iterative development of scales was designed based upon a number of 23 proposed metrics for measuring the effectiveness of mobile application. These metrics (measured and expressed in units) were collected and gathered by considering multiple theories to integrate both objective and subjective measures 
for effectiveness evaluation. The original metrics were modified to address the requirements for assessing the importance of measuring the effectiveness of handheld-based application system and specific user tasks. For example, to modify the effectiveness metric into question, 'number of skills integrated correctly'. Thus result, 'I think, it is important to measure the number of skills integrated correctly within session or treatment' question. This scales consisted of 23 items rated on a five-point Likert scale from extremely agree, slightly agree, neutral, slightly disagree and extremely disagree. The modification of the effectiveness measurement item is as shown in Table 2 above.

A total number of 397 targeted participants responded. After exclusion of duplicate entries and missing entries (more than 3.27\% of incomplete data), there were 384 valid responses. This study used list wise deletion for missing and duplicate data, therefore only valid responses were used. The perceived mobile usage competency of the respondents was high. Results reported more than $50 \%$ of respondents somewhat agreeing, strongly agreeing and extremely agreeing that they were competent.

\subsection{Weightage Extraction and Code Specificity}

Table 3. Weightage Value and Code Specificity

\begin{tabular}{|c|c|c|c|c|}
\hline Criterion & Attribute & Metric & Weightage & Code \\
\hline \multirow[t]{13}{*}{ Accurateness } & & & .381 & $w_{E F F I}$ \\
\hline & Action Adequacy & & .322 & $w_{A C C l}$ \\
\hline & & Error Prevented & .446 & $w_{A A I}$ \\
\hline & & Error Detected & .401 & $w_{A A 2}$ \\
\hline & & Failure Recovered & 444 & $w_{A A 3}$ \\
\hline & Literacy Error & & 380 & $w_{A C C 2}$ \\
\hline & & Text Read & .501 & $w_{L E I}$ \\
\hline & & Syllable Spoken & .400 & $w_{L E 2}$ \\
\hline & & Word Written & .331 & $w_{L E 3}$ \\
\hline & Spatial Accuracy & & .404 & $w_{A C C 3}$ \\
\hline & & Target Identified & .364 & $w_{S A I}$ \\
\hline & & Input Entered & 286 & $w_{S A 2}$ \\
\hline & & Page Navigated & .414 & $w_{S A 3}$ \\
\hline \multirow[t]{14}{*}{ Readiness } & & & .368 & $w_{E F F 2}$ \\
\hline & Knowledge Acquisition & & .483 & $w_{R D N 1}$ \\
\hline & & Skill Integrated & .580 & $w_{K A I}$ \\
\hline & & Problem Solved & .527 & $w_{K A 2}$ \\
\hline & & Meaning Comprehended & .564 & $w_{K A 3}$ \\
\hline & & Reason Explained & 463 & $w_{K A 4}$ \\
\hline & Individual Interpretation & & .395 & $w_{R D N 2}$ \\
\hline & & Answer Supplied & .553 & $w_{I I I}$ \\
\hline & & Example Provided & .579 & $w_{I I 2}$ \\
\hline & & Question Understood & .622 & $w_{I I 3}$ \\
\hline & Interface Acceptance & & .454 & $w_{R D N 3}$ \\
\hline & & Information Memorized & .388 & $w_{I A I}$ \\
\hline & & Representations Recalled & .442 & $w_{I A 2}$ \\
\hline & & Layouts Recognized & .309 & $w_{\text {IAS }}$ \\
\hline \multirow[t]{7}{*}{ Completeness } & & & .304 & $w_{\text {EFF3 }}$ \\
\hline & Goal Accomplishment & & .350 & $w_{C M P I}$ \\
\hline & & Task Completed & .512 & $w_{G A I}$ \\
\hline & & Task Completed $(\mathrm{T})$ & .311 & $w_{G A 2}$ \\
\hline & User Willingness & & .390 & $w_{C M P 2}$ \\
\hline & & Self-Motivated & .349 & $w_{U W I}$ \\
\hline & & Self-Motivated (T) & .444 & $w_{U W 2}$ \\
\hline
\end{tabular}

Weightage extraction was performed based on previous work regarding the identification and determination of measures for assessing the effectiveness of mobile applications. The scale was used to 
gather information from respective users to indicate their level of agreement towards the importance of each effectiveness measure, based on their experience and perception. Data collected were entered into the statistical software program for analysis. Relationship evaluation test was carried out in the software program to determine the strength between measures in different hierarchical levels in order to assess the overall effectiveness.

Formula for calculating the effectiveness of mobile applications could also be constructed by applying weights. Weight values were coded either as $W_{A T T m}$ for representing weight value of metric, or $W_{\text {CRTa }}$ for representing weight value of attribute or $W_{E F F C}$ for representing weight value of criterion. The generic symbol $W_{\text {ATTm }}$ represents the weight code of metric mth that contributes towards its corresponding attribute ATT. Meanwhile, symbol $W_{C R T a}$ represents the weight code of attribute ath that contributes towards its corresponding criterion CRT. Finally, symbol $W_{E F F c}$ represents the weight code of criterion cth that contributes towards measuring the overall effectiveness of mobile applications, EFF. The code specificity for each metric, attribute and criterion for measuring the effectiveness of mobile applications is shown in Table 3 above.

\subsection{Optimisation of Parameters}

Table 4. Codes of Effectiveness Parameters

\begin{tabular}{|c|c|c|c|}
\hline Criterion & Attribute & Metric & Linearity Code \\
\hline \multirow[t]{13}{*}{ Accurateness } & & & $C_{l} \bullet F_{E F F}$ \\
\hline & Action Adequacy & & $A_{l} \bullet C_{l} \bullet F_{E F F}$ \\
\hline & & Error Prevented & $M_{l} \bullet A_{l} \bullet C_{l} \bullet F_{E F F}$ \\
\hline & & Error Detected & $M_{2} \bullet A_{l} \bullet C_{l} \bullet F_{E F F}$ \\
\hline & & Failure Recovered & $M_{3} \bullet A_{l} \bullet C_{I} \bullet F_{E F F}$ \\
\hline & Literacy Error & & $A_{2} \bullet C_{l} \bullet F_{E F F}$ \\
\hline & & Text Read & $M_{1} \bullet A_{2} \bullet C_{1} \bullet F_{E F F}$ \\
\hline & & Syllable Spoken & $M_{2} \bullet A_{2} \bullet C_{l} \bullet F_{E F F}$ \\
\hline & & Word Written & $M_{3} \bullet A_{2} \bullet C_{1} \bullet F_{E F F}$ \\
\hline & Spatial Accuracy & & $A_{3} \bullet C_{l} \bullet F_{E F F}$ \\
\hline & & Target Identified & $M_{1} \bullet A_{3} \bullet C_{l} \bullet F_{E F F}$ \\
\hline & & Input Entered & $M_{2} \bullet A_{3} \bullet C_{l} \bullet F_{E F F}$ \\
\hline & & Page Navigated & $M_{3} \bullet A_{3} \bullet C_{l} \bullet F_{E F F}$ \\
\hline \multirow[t]{14}{*}{ Readiness } & & & $C_{2} \bullet F_{E F F}$ \\
\hline & Knowledge Acquisition & & $A_{1} \bullet C_{2} \bullet F_{E F F}$ \\
\hline & & Skill Integrated & $M_{1} \bullet A_{l} \bullet C_{2} \bullet F_{E F F}$ \\
\hline & & Problem Solved & $M_{2} \bullet A_{1} \bullet C_{2} \bullet F_{E F F}$ \\
\hline & & Meaning Comprehended & $M_{3} \bullet A_{1} \bullet C_{2} \bullet F_{E F F}$ \\
\hline & & Reason Explained & $M_{4} \bullet A_{l} \bullet C_{2} \bullet F_{E F F}$ \\
\hline & Individual Interpretation & & $A_{2} \bullet C_{2} \bullet F_{E F F}$ \\
\hline & & Answer Supplied & $M_{1} \bullet A_{2} \bullet C_{2} \bullet F_{E F F}$ \\
\hline & & Example Provided & $M_{2} \bullet A_{2} \bullet C_{2} \bullet F_{E F F}$ \\
\hline & & Question Understood & $M_{3} \bullet A_{2} \bullet C_{2} \bullet F_{E F F}$ \\
\hline & Interface Acceptance & & $A_{3} \bullet C_{2} \bullet F_{E F F}$ \\
\hline & & Information Memorized & $M_{1} \bullet A_{3} \bullet C_{2} \bullet F_{E F F}$ \\
\hline & & Representations Recalled & $M_{2} \bullet A_{3} \bullet C_{2} \bullet F_{E F F}$ \\
\hline & & Layouts Recognized & $M_{3} \bullet A_{3} \bullet C_{2} \bullet F_{E F F}$ \\
\hline \multirow[t]{7}{*}{ Completeness } & & & $C_{3} \bullet F_{E F F}$ \\
\hline & Goal Accomplishment & & $A_{l} \bullet C_{3} \bullet F_{E F F}$ \\
\hline & & Task Completed & $M_{1} \bullet A_{l} \bullet C_{3} \bullet F_{E F F}$ \\
\hline & & Task Completed (T) & $M_{2} \bullet A_{l} \bullet C_{3} \bullet F_{E F F}$ \\
\hline & User Willingness & & $A_{2} \bullet C_{3} \bullet F_{E F F}$ \\
\hline & & Self Motivated & $M_{1} \bullet A_{2} \bullet C_{3} \bullet F_{E F F}$ \\
\hline & & Self Motivated (T) & $M_{2} \bullet A_{2} \bullet C_{3} \bullet F_{E F F}$ \\
\hline
\end{tabular}


Lists of codes were produced to represent each effectiveness metric, attribute and criterion, presented as $M_{m} \bullet A_{a} \bullet C_{c} \bullet F_{E F F}, A_{a} \bullet C_{c} \bullet F_{E F F}$, and $C_{c} \bullet F_{E F F}$, respectively. $M$ represents metric, meanwhile $A$ represents attribute, and $\mathrm{C}$ represents criterion, whereas $\mathrm{F}$ represents effectiveness as a factor for assessing mobile applications. Based on the rank order for each metric towards its corresponding attribute, presented as $M_{m} \bullet A_{a} \bullet C_{c} \bullet F_{E F F}$, m represents the sequential series (m-th) of the metric, such as 1, 2, ..., m, that contributed towards a particular attribute, a. In addition, presented as $A_{a} \bullet C_{c} \bullet F_{E F F}$, a represents the sequential series (ath) of the attribute, such as $1,2, \ldots$, a, that contributed towards particular criterion, c. Finally, presented as $C_{c} \bullet F_{E F F}, \mathrm{c}$ represents the sequential series (c-th) of the criterion, such as $1,2, \ldots, \mathrm{c}$, that contributed towards effectiveness as the factor for assessing the mobile applications, in which EFF represents the abbreviation of Effectiveness. The linearity code for each metric, attribute and criterion for measuring the effectiveness of mobile applications is shown in Table 4 above.

\subsection{Mathematical Equations}

An equation of effectiveness metric was formulated to determine the relative quantification of a target activity in comparison to a reference activity. The effectiveness metric expression ratio ( $M_{m} \bullet A_{a} \bullet$ CRDN) of a target activity is calculated based on the number of activities performed correctly (Etarget), where the deviation is the difference between an actual activity and an expected activity ( $\Delta$ target (actual - expected)). This was expressed in comparison to a reference activity calculated based on the total number of activities performed (Ereference) and the total number of expected activities (Treference (expected)).

Equation 1 shows a mathematical model of relative expression ratio in quantifying effectiveness metrics. The ratio is expressed as an expected versus actual target activity, in comparison to a reference expected activity. E target is the observed effectiveness metric of target activity transcript, E reference is the observed effectiveness metric of reference activity transcript, $\Delta$ target is the deviation of actual - expected of the target activity transcript, and $T$ reference is the total of expected reference activity transcript. The expected activity could be a constant and a regulated transcript, which means that for the calculation of effectiveness metric ratio $\left(M_{m} \bullet A_{a} \bullet \mathrm{CRDN}\right)$, the individual target expected activity, target(expected) and the reference expected activity, reference(expected) of the investigated transcript must be known, and only dependent on the target actual activity target(actual).

$$
\frac{\left(E_{\text {target }}\right){ }^{\Delta \text { target (actual - expected })}}{\left(E_{\text {reference }}\right)^{\text {Treference (expected })}}
$$

An equation for assessing effectiveness attributes of mobile applications was formulated by determining the relative summation of the product of weight and value in comparison to the average of weight. In detail, the attribute expression ratio $\left(A_{a} \bullet C_{C} \bullet F_{E F F}\right)$ of mobile applications effectiveness is calculated based on the total product of each metric weightage $\left(W_{A T T m}\right)$ multiplied by the corresponding metric values $\left(M_{m} \bullet A_{a} \bullet C_{c} \bullet F_{E F F}\right)$, and expressed in comparison to the average weightage of metric $\left(W_{A T T m}\right)$. Equation 2 shows a mathematical model of relative expression ratio in quantifying effectiveness attributes of mobile applications.

$$
\begin{array}{ll}
\sum_{m=1}^{m} w_{A T T m}\left(M_{m} \bullet A_{a} \bullet C_{c} \bullet F_{E F F}\right) \\
\hline m \\
\sum_{m=1} w_{A T T m}\left(M_{m} \bullet A_{a} \bullet C_{c} \bullet F_{E F F}\right)
\end{array}
$$


Meanwhile, an equation for assessing effectiveness criterion of mobile applications was formulated by determining the relative summation of the product of weight and value in comparison to the average of weight. In detail, the criterion expression ratio $\left(C_{c} \bullet F_{E F F}\right)$ of mobile applications effectiveness is calculated based on the total product of each attribute weightage $\left(W_{A T T a}\right)$ multiplied by the corresponding attribute values $\left(A_{a} \bullet C_{c} \bullet F_{E F F}\right)$, and expressed in comparison to the average weightage of attribute ( $\left.W_{A T T a}\right)$. Equation 3 shows a mathematical model of relative expression ratio in quantifying effectiveness criterions of mobile applications.

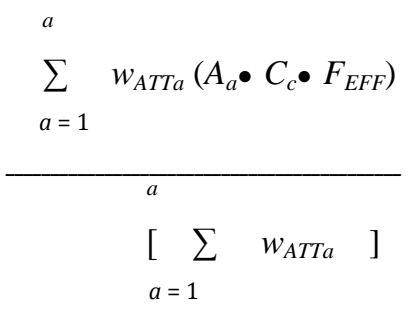

Finally, an equation for assessing the total amount of mobile applications effectiveness $\left(F_{E F F}\right)$, was formulated based on the total product of each criterion weightage $\left(W_{A T T C}\right)$ multiplied by the corresponding criterion values $\left(C C_{\bullet} F_{E F F}\right)$, and expressed in comparison to the average weightage of criterion ( $\left.W_{A T T C}\right)$. Equation 4 shows a mathematical model of relative expression ratio in quantifying effectiveness of mobile applications.

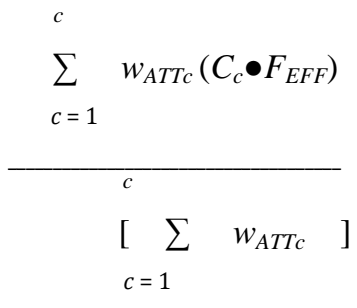

\section{Mobile Applications Effectiveness Measurement Model}

As a result of these quantification methods, a model, namely Mobile Applications Effectiveness Measurement Model (MoAEMM), has been proposed which suggests how mobile applications effectiveness should be evaluated. The model is organized by metrics, attributes, criterions and effectiveness as the factor for assessing mobile applications. For each attribute, the model describes relevant effectiveness metrics appropriate for measurement and potential evaluation measures. The classification scheme in Fig. 2 summarizes the construct and the measures proposed throughout this research, and advance the effectiveness evaluation by providing a quantitative approach in assessing the general effectiveness of mobile applications.

The existence of interrelations between metrics and attributes should be taken into account in determining the level of effectiveness of mobile applications. Due to the linear and hierarchical structure of the MoAEMM, any changes to metrics will result in changes to the attributes and consequently on the overall effectiveness of the mobile applications usage. For example, a low score on the matric (i.e. Skills Integrated $\left(M_{1} \bullet A_{1} \bullet C_{2} \bullet F_{E F F}\right)$ ) will directly affect the score of the attribute Knowledge Acquisition $\left(A_{1} \bullet C_{2} \bullet F_{E F F}\right)$ and results in significant implications for the overall effectiveness $\left(F_{E F F}\right)$ of mobile applications usage, and vice versa.

However, to obtain the precise numeric value is as tangible as the likelihood of occurrences is impossible. Fortunately, exact figures for measuring effectiveness are not needed since the numbers are mostly used for comparison purpose only. Thus, prioritizing the effectiveness can be done by converting the values into 
words or sentences with which the evaluator from various background and understanding can interpret the information accurately and comprehensively.

Prioritizing overall effectiveness usage can be categorized into five distinct classifications (refer Table 5). The lowest level indicates the most badly absence or shortage of a desirable usage effectiveness whilst the highest level represents outstanding or fulfilment of a desirable usage effectiveness with high distinction of proficiency. It is important to note that prioritizing the level for measuring the effectiveness of mobile applications usage mentioned above is flexible and does not fixed to the stated figures. The scores for each level are open for customization and tailored to specific requirements according to the maturity of the mobile applications itself or based on the evaluator's wishes.

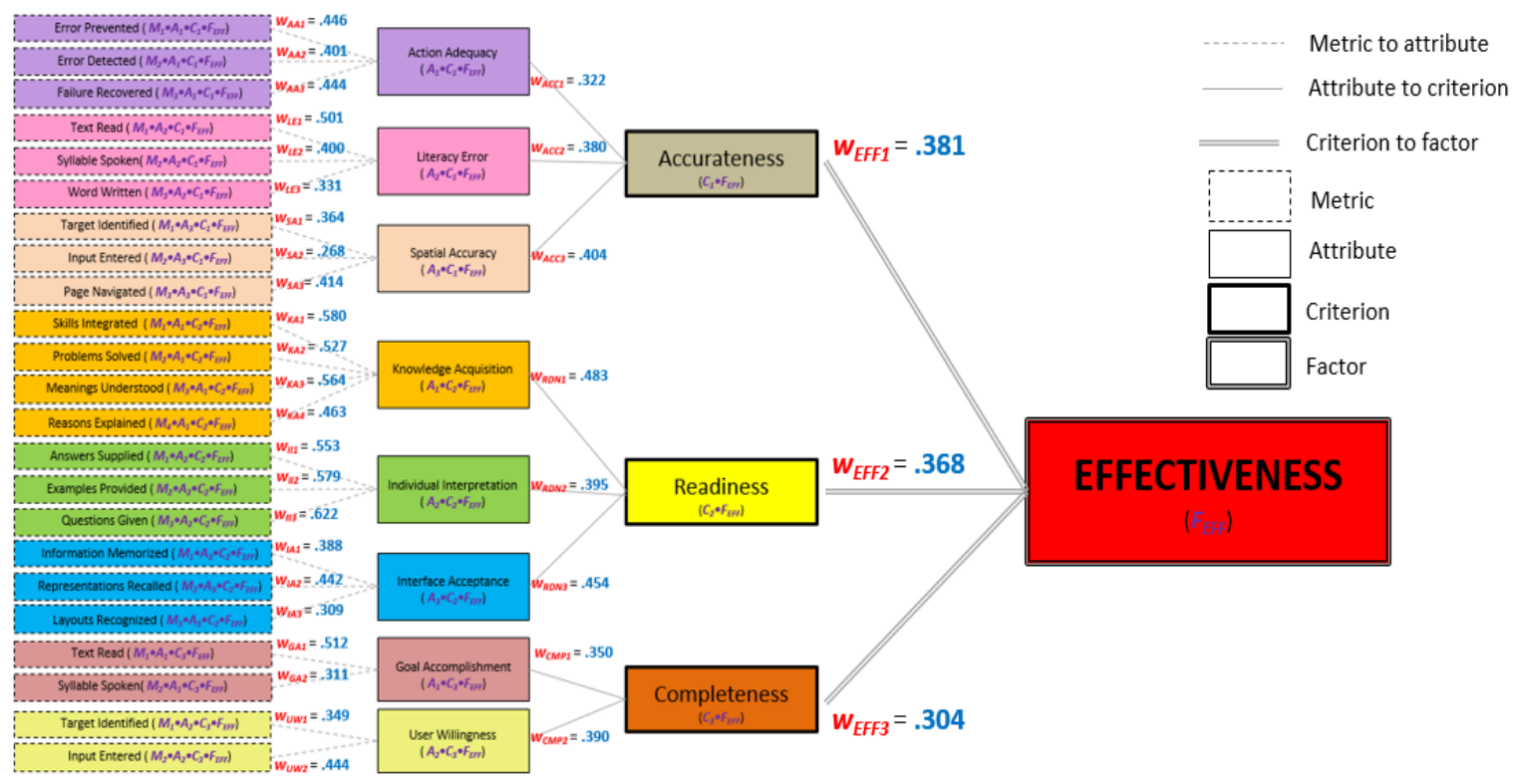

Fig. 2. Mobile applications effectiveness measurement model (MoAEMM).

Table 5. Prioritizing Overall Effectiveness

\begin{tabular}{|c|c|c|l|}
\hline Score & Level & Status & Description \\
\hline $\mathrm{F}_{\mathrm{EFF}}<0.200$ & 1 & Worst & $\begin{array}{l}\text { Most badly absence or shortage of a desirable usage effectiveness that results } \\
\text { users unable to perform comprehensively }\end{array}$ \\
\hline $\mathrm{F}_{\mathrm{EFF}}<0.400$ & 2 & Inadequate & $\begin{array}{l}\text { Lack of a desirable usage effectiveness that results users with the least } \\
\text { excellent to perform task }\end{array}$ \\
\hline $\mathrm{F}_{\mathrm{EFF}}<0.600$ & 3 & Acceptable & $\begin{array}{l}\text { Average of a desirable usage effectiveness that can be tolerable to be } \\
\text { considered as good enough }\end{array}$ \\
\hline $\mathrm{F}_{\mathrm{EFF}}<0.800$ & 4 & Excellent & $\begin{array}{l}\text { Complete the specific requirements of a desirable usage effectiveness that } \\
\text { achieves almost in a state of being practical }\end{array}$ \\
\hline $\mathrm{F}_{\mathrm{EFF}} \leq 1.000$ & 5 & Outstanding & $\begin{array}{l}\text { Fulfilment of all requirements of desirable usage effectiveness that achieves } \\
\text { very high distinction of proficiency }\end{array}$ \\
\hline
\end{tabular}

\section{Conclusion}

Both the theory and practice of quantifying mobile applications effectiveness have been hampered by the absence of a thorough mathematically based model as a method for evaluation. As a result, this research effort has been in a position to derive a preliminary mathematically based specification and measurement scheme specifically for assessing the mobile applications effectiveness. The ultimate value for developing a mathematical oriented approach is to provide a systematic and quantitative method for conducting mobile applications effectiveness evaluation research. 


\section{Acknowledgment}

My thanks to Faculty of Science and Defence Technology, National Defence University of Malaysia, Centre of Research and Innovation, National Defence University of Malaysia, and Ministry of Higher Education, Malaysia

\section{References}

[1] Bevan, N., \& Macleod, M. (1994). Usability measurement in context. Behaviour and Information Technology, 13, 132-145.

[2] Frojkaer, E., Hertzum, M. \& Hornbaek, K. (2000). Measuring usability: Are effectiveness, efficiency, and satisfaction really correlated. $\mathrm{CHI}^{\prime} 00,345-352$. New York: ACM Press.

[3] ISO 9241-11. (1998). Ergonomic requirements for office work with visual display terminals (VDTs). Guidance on Usability.

[4] Dubey, S. K., \& Rana, A. (2010). Analytical roadmap to usability definitions and decompositions. International Journal of Engineering Science and Technology, 2(9), 4723-4729.

[5] Greenspun, H., \& Coughlin, S. (2012). mHealth in an mWorld: How mobile technology is transforming health care. Deloitte Center for Health Solutions.

[6] Free, C., Phillips, G., Watson, L., Galli, L., Felix, L., \& Edwards, P. (2013). The effectiveness of mobile-health technologies to improve health care service delivery processes: A systematic review and meta-analysis. PLoS Med, 10(1).

[7] Gary, A. (2011). Invasion of the mobile apps. Communications of the ACM, 54(9), 16-18.

[8] Dehlinger, J., \& Dixon, J. (2011). Mobile application software engineering: Challenges and research directions. Proceedings of the Workshop on Mobile Software Engineering (pp. 29-32).

[9] Zhang, D., Adipat, B. (2005). Challenges, methodologies, and issues in the usability testing of mobile applications. International Journal of Human-Computer Interaction, 18(3), 293-308.

[10] Gafni, R. (2009). Usability issues in mobile - wireless information systems. Issues in Informing Science and Information Technology, 6, 755-769.

[11] Yahaya, J., Deraman, A., Baharom, F., \& Hamdan, A. R. (2011). SPQF: Software process quality factor: For software process assessment and certification. Proceedings of the International Conference on Electrical Engineering and Informatics.

[12] Deraman, A., Kamaruddin, A., Yahya, S., Selamat, H., \& Zulzalil, H. (2001). Perception on software certification model: An empirical study. Proceeding of the International Conferencen on Information Technology and Multimedia (pp. 464-469).

[13] Fadzlah, A. \& Deraman, A. (2007). Measuring the usability of software applications: Metrics for behaviorness. Proceedings of the International Conference on Computational Science and Its Applications: Vol 2.

[14] Fadzlah, A. \& Deraman, A. (2007). Measuring the usability of software applications: Metrics for accurateness. Proceedings of the International Conference on Electrical Engineering and Informatics.

[15] Fadzlah, A. (2016). Defence mobile application system usability evaluation scale (Defence-MoASUES): Examining construct validity using exploratory factor analysis results. Proceedings of the International Conference on Defence and Security Technology (DSTC2016).

[16] Paz, F., \& Pow-Sang, J. A. (2014). Current trends in usability evaluation methods: A systematic review. Proceedings of the 7th International Conference on Advanced Software Engineering and Its Applications (pp. 11-15).

[17] Nielsen, J. \& Landauer, T. K. (1993). A mathematical model of the finding of usability problems. Proceedings of the INTERACT '93 and CHI '93 Conference on Human Factors in Computing Systems (pp. 206- 
213).

[18] Ghasemifard, N., Shamsi, M., Kenari, A. R., \& Ahmadi, V. (2015). A new view at usability test methods of interfaces for human computer interaction. Global Journal of Computer Science and Technology, 15(1).

[19] Fadzlah, A. (2011). An initial mathematical model for quantifying the accuracy of handheld application usage. Proceedings of the International Conference on Computer and Software Modeling.

[20] Fadzlah, A. (2016). Readiness measurement model (RMM): Mathematical-based evaluation technique for the quantification of knowledge acquisition, individual understanding, and interface acceptance dimensions of software applications on handheld devices. Proceedings of the 4th International Conference on Computer Science, Applied Mathematics and Applications.

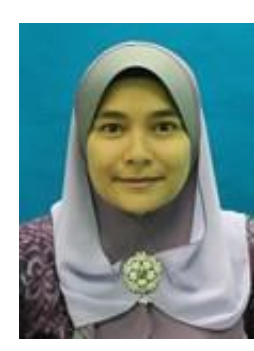

Amalina F. A. Fadzlah obtained the bachelor of information technology degree in networking (with honours) in 1998 and the master of science in information technology degree in 2002 from the Faculty of Information Technology, Northern University of Malaysia. She also obtained the doctor of philosophy degree in computer science in 2010 from the Faculty of Technology and Information Science, National University of Malaysia. Currently, she is a senior lecturer at the Department of Computer Science, Faculty of Defence Science and Technology, National Defence University of Malaysia.

She has more than fifteen years of extensive experience in teaching and mentoring students, and comprehensive experience in the research and development of mobile-based software application systems, with an interest in the field of software engineering specializing in software quality, software certification, software management, and human computer interaction. 